\title{
The cell adhesion molecule TMIGD1 binds to moesin and regulates tubulin acetylation and cell migration
}

\author{
Nader Rahimi ${ }^{*}{ }^{*}$, Rachel X. Y. Ho ${ }^{1}$, Kevin Brown Chandler ${ }^{2}$, Kyle Oliver Corcino De La Cena ${ }^{1}$, Razie Amraei ${ }^{1}$, \\ Ashley J. Mitchel ${ }^{1}$, Nels Engblom ${ }^{1 *}$ and Catherine E. Costello ${ }^{2^{*}}$
}

\begin{abstract}
Background: The cell adhesion molecule transmembrane and immunoglobulin (lg) domain containing1 (TMIGD1) is a novel tumor suppressor that plays important roles in regulating cell-cell adhesion, cell proliferation and cell cycle. However, the mechanisms of TMIGD1 signaling are not yet fully elucidated.

Results: TMIGD1 binds to the ERM family proteins moesin and ezrin, and an evolutionarily conserved RRKK motif on the carboxyl terminus of TMIGD1 mediates the interaction of TMIGD1 with the N-terminal ERM domains of moesin and ezrin. TMIGD1 governs the apical localization of moesin and ezrin, as the loss of TMIGD1 in mice altered apical localization of moesin and ezrin in epithelial cells. In cell culture, TMIGD1 inhibited moesin-induced filopodia-like protrusions and cell migration. More importantly, TMIGD1 stimulated the Lysine (K40) acetylation of a-tubulin and promoted mitotic spindle organization and CRISPR/Cas9-mediated knockout of moesin impaired the TMIGD1-mediated acetylation of a-tubulin and filamentous (F)-actin organization.
\end{abstract}

Conclusions: TMIGD1 binds to moesin and ezrin, and regulates their cellular localization. Moesin plays critical roles in TMIGD1-dependent acetylation of a-tubulin, mitotic spindle organization and cell migration. Our findings offer a molecular framework for understanding the complex functional interplay between TMIGD1 and the ERM family proteins in the regulation of cell adhesion and mitotic spindle assembly, and have wide-ranging implications in physiological and pathological processes such as cancer progression.

Keywords: TMIGD1, Moesin, Ezrin, ERM family proteins, Tubulin acetylation, Mitotic spindle, Microtubular, Tumor suppressor, Cell migration

\section{Background}

Altered cell-cell adhesion and disruption of apico-basal polarity are common features of all carcinomas [14, 39]. Tumor suppressor genes such as $V H L$ and $A P C$ that are frequently inactivated in human renal and colon cancers, are also key regulators of cell adhesion and polarity[13,

\footnotetext{
*Correspondence: nrahimi@bu.edu; cecmsms@bu.edu

${ }^{1}$ Department of Pathology, School of Medicine, Boston University

Medical Campus, Boston, MA 02118, USA

${ }^{2}$ Center for Biomedical Mass Spectrometry, Boston University School

of Medicine, Boston, MA 02118, USA
}

23]. First discovered as a regulator of cell-cell adhesion and cell morphology, the cell adhesion molecule transmembrane and immunoglobulin (Ig) domain containing-1 (TMIGD1) is predominantly expressed in kidney and intestinal epithelial cells and protects renal epithelial cells from oxidative cell injury, thus promoting cell survival [2]. Emerging evidence on the role of TMIGD1 in human cancers points to TMIGD1 as a novel tumor suppressor. TMIGD1 is downregulated in human renal and colon cancers [10, 27]. Re-expression of TMIGD1 in renal and colon cancer cell lines inhibits cell proliferation and induces $\mathrm{G} 2 / \mathrm{M}$ cell cycle checkpoint arrest [10, original author(s) and the source, provide a link to the Creative Commons licence, and indicate if changes were made. The images or other third party material in this article are included in the article's Creative Commons licence, unless indicated otherwise in a credit line to the material. If material is not included in the article's Creative Commons licence and your intended use is not permitted by statutory regulation or exceeds the permitted use, you will need to obtain permission directly from the copyright holder. To view a copy of this licence, visit http://creativecommons.org/licenses/by/4.0/. The Creative Commons Public Domain Dedication waiver (http://creativeco mmons.org/publicdomain/zero/1.0/) applies to the data made available in this article, unless otherwise stated in a credit line to the data. 
27]. Recent studies have revealed that loss of TMIGD1 in mice significantly impairs intestinal epithelium brush border membrane junctional polarity and maturation, resulting in the development of adenomas in small intestine and colon [10]. TMIGD1 inhibits tumor cell proliferation and cell cycle arrest at the G2/M phase through regulating expression of $\mathrm{p}_{21}{ }^{\mathrm{CIP} 1}$ (cyclin-dependent kinase inhibitor 1), and $\mathrm{p} 27^{\mathrm{KIP1}}$ (cyclin-dependent kinase inhibitor 1B) [10]. However, the specific mechanisms by which TMIGD1 elicits these effects, particularly at the level of protein-protein interaction remain unknown. TMIGD1 is a transmembrane glycoprotein that consists of an extracellular domain with two Ig domains, a single transmembrane domain, and a highly conserved short intracellular domain enriched in the positively charged amino acids Lysine (K) and Arginine (R)[2], with a potential to recruit signaling proteins to TMIGD1 [2].

Ezrin, radixin and moesin (ERM), three highly similar proteins are members of the FERM (4.1-band ERM) superfamily, which are central for linking the actin cytoskeleton to the cell membrane and are major regulators of specialized membrane domains, including apical microvilli [22, 38], lamellipodia and filopodia [4, 21]. Due to their function as cytoskeletal linkers, ERM family proteins play essential roles in diverse cellular processes ranging from cell-cell adhesion and cell migration to cell proliferation [8], and thus play significant roles in the metastatic progression of human cancers. Furthermore, the FERM protein moesin is known to bind to microtubules (MTs) and mediate the association of actin filaments, which is required for regulating spindle organization during mitosis $[36,41]$. MTs are cytoskeletal filaments composed of heterodimers of $\alpha$ - and $\beta$-tubulin subunits and are critically important for chromosomal segregation, intracellular transport, cell division, cell motility and cell morphogenesis [30]. Post translational acetylation of tubulin stabilizes MTs and plays a central role in its dynamic features and cellular functions, a process exploited by anti-tumor agents such as Taxol to promote mitotic arrest and cell death [42]. Here, we present evidence that TMIGD1 binds to ERM family proteins (moesin and ezrin), regulates the stability of microtubules and modulates cell migration in renal cancer cells, implicating TMIGD1 as a potential cancer therapeutic target.

\section{Methods}

\section{Plasmids and antibodies}

Moesin-GFP, ezrin-GFP, GST-FERM-moesin, GST-CERMAD-moesin, and LifeAct7 plasmids were purchased from Addgene. Moesin sgRNA was purchased from Dharmacon. Construction and cloning of TMIGD1 into retroviral, pMSCV.puro vector was previously described
[2, 27]. GST-TMIGD1 and GST-4A-TMIGD1 were generated by standard PCR amplification of the cytoplasmic region of TMIGD1 and cloned into pGEX-4T-2 vector. Plasmids were expressed in E.coli and GST-fusion proteins were purified from E.coli via glutathione Sepharose Fast-flow resin per manufacturer's recommendations. Polyclonal rabbit anti- $\alpha$-tubulin antibody, polyclonal rabbit-anti-acetyl (K40)-tubulin antibody, anti-Myc antibody, and anti-GFP antibody all were purchased from Cell Signaling Technology Inc., (Danvers, MA). Development and characterization of the polyclonal anti-TMIGD1 antibody used in these studies has been described previously [2].

\section{Cell culture}

HEK-293, RKO, 786-0 cells were each maintained in Dulbecco's modified Eagle medium (DMEM) supplemented with $10 \%$ fetal bovine serum (FBS), L-glutamine $(2 \mathrm{mM})$, penicillin (50 units/ml) and streptomycin $(50 \mathrm{mg} / \mathrm{ml})$.

\section{Animal studies}

CRISPR/Cas9-mediated TMIGD1 knockout mice were developed in our laboratory [10]. PFA fixed tissues were stained for moesin, ezrin or for a protein of interest, as described in the figure legends.

\section{Retrovirus production and transfection}

pMSCV.puro vector containing TMIGD1 or another cDNA of interest was transfected into 293-GPG cells, and viral supernatants were collected for five days as previously described [32] and viral supernatants were used to generate stable RKO or HEK-293 cell lines expressing TMIGD1 or empty vector (EV) as a control. TMIGD1 expressing cells were selected with puromycin. In some experiments as described in the figure legends, these cell lines were transiently transfected with moesin-GFP, ezrin-GFP or other plasmids via PEI (polyethylenimine). After $48 \mathrm{~h}$, cells were lysed and subjected to co-immunoprecipitation or western blotting as described in the figure legends.

\section{Immunoprecipitation and western blot analysis}

Cells were grown in 10-cm culture dishes until 80 to $90 \%$ confluence. Cells were lysed, and normalized whole-cell lysates were subjected to immunoprecipitation by incubation with appropriate antibodies as shown in the figure legends. Immuno-complexes were captured by incubation with either protein A-Sepharose or protein G-agarose beads. After release via boiling the samples for $5 \mathrm{~min}$ at $95{ }^{\circ} \mathrm{C}$, the immunoprecipitated proteins were subjected to western blot analysis. Occasionally, membranes were stripped by incubating them in a stripping buffer containing $6.25 \mathrm{mM}$ Tris- $\mathrm{HCl}, \mathrm{pH} 6.8,2 \%$ SDS, and 
$100 \mathrm{mM} \beta$-mercaptoethanol at $50{ }^{\circ} \mathrm{C}$ for $30 \mathrm{~min}$, washed in Western Rinse buffer $(20 \mathrm{mM}$ Tris and $150 \mathrm{mM} \mathrm{NaCl})$, and re-probed with the antibody of interest. The blots were scanned and subsequently quantified using Image J (NIH).

\section{GST-pulldown assay}

In vitro GST fusion protein binding experiments were performed as described previously [26]. Briefly, equal numbers of cells expressing TMIGD1 or other proteins, as described in the figure legends were grown to $90 \%$ confluence. Cells were lysed in ice-cold lysis buffer supplemented with $2 \mathrm{mM} \mathrm{Na}_{3} \mathrm{VO}_{4}$ and a protease inhibitor cocktail. Equal amounts of the appropriate immobilized GST fusion proteins were incubated with normalized whole-cell lysates by rocking for $3 \mathrm{~h}$ at $4{ }^{\circ} \mathrm{C}$. The beads were washed in the presence of protease inhibitors, and proteins were eluted and analyzed by western blotting using the appropriate antibody, as described in the figure legends.

\section{Liquid chromatography-tandem mass spectrometry (LC- MS/MS)}

Agarose resins with GST-TMIGD1 bound via glutathione were incubated with whole cell lysate derived from 786-0 cells. Captured proteins were resolved on SDSPAGE. Bands of interest were excised from the gel, and individual bands were subjected to trypsin digestion (at $37^{\circ} \mathrm{C}$ overnight in $50 \mathrm{mM}$ ammonium bicarbonate). Peptides were separated and analyzed via a 6550 Q-TOF MS equipped with a 1200 series nanoflow HPLC-Chip ESI source fitted with an HPLC-Chip consisting of a 360-nl trapping column and a $150 \mathrm{~mm} \times 75 \mu \mathrm{m}$ analytical column, both packed with Polaris C18-A 3- $\mu$ m material (all from Agilent Corp.). After injection of the sample onto the trapping column, the column was washed at a rate of $2 \mu \mathrm{l} / \mathrm{min}$ with $2 \%$ acetonitrile and $0.1 \%$ formic acid in water for $4 \mathrm{~min}$. Peptides were then separated on the analytical column at a flow rate of $0.3 \mu \mathrm{l} / \mathrm{min}$ using a gradient from 2 to $40 \%$ acetonitrile with $0.1 \%$ formic acid over $25 \mathrm{~min}$. The 6550 Q-TOF mass spectrometer was operated in the positive-ion mode using the high-resolution, extended dynamic range $(2 \mathrm{GHz})$ setting. The instrument was operated in data-dependent mode; the 20 most abundant ions were selected for MS/MS. MS spectra were recorded over the range $m / z 285-1700$, and MS2 spectra were recorded from $m / z 50-3000$. The ion source gas temperature was set to $225^{\circ} \mathrm{C}$, and the flow was set at $13 \mathrm{l} / \mathrm{min}$, with a capillary voltage of $2100 \mathrm{~V}$. Precursors $\geq 5000$ counts and charge states $\geq 2$ were selected for fragmentation, and the collision energy was set according to the equation $y=m x+b$, with $y$ being the collision energy, slope $m=3.6, x$ representing the charge state, and the offset $b=-4.8$ for charge states 3 and 4 . For charge state 2 peptides, slope $m=3.1$, and the offset $b=1$. Spectra were recorded in the centroid mode. MS/MS spectra were searched using a local copy of Mascot (www.matri xscience.com) using the Uniprot database of human proteins. The selected parameters required a minimum of two peptide matches per protein, with minimum probabilities of $95 \%$ at the peptide level.

\section{Immunofluorescence microscopy}

Cells expressing TMIGD1 alone or together with moesin were seeded onto coverslips and grown overnight in $60-\mathrm{mm}$ plates to $80-100 \%$ confluence. The cells were washed once with PBS and fixed with freshly prepared $4 \%$ paraformaldehyde for $15 \mathrm{~min}$ at room temperature. After being washed three more times with PBS, the cells were permeabilized with $0.25 \%$ Triton X-100 in Western rinse for $10 \mathrm{~min}$ at room temperature and then washed three times with PBS. For staining with anti- $N$-acetyl tubulin or total tubulin, the cells were blocked with (1:1) BSA in Western Rinse buffer for $1 \mathrm{~h}$ and washed once in PBS, followed with incubation with the selected antibody, as indicated in the figure legends, for $1 \mathrm{~h}$ and then detected with a FITC-conjugated secondary antibody. The coverslips were mounted in Vectashield mounting medium with DAPI onto glass microscope slides. The slides were analyzed under a fluorescence microscope.

\section{F-actin stress orientation quantification}

786-0 cells expressing TMIGD1 alone or with other constructs were stained for actin, as described above, and visualized using an inverted epifluorescence microscope. From each image, five different regions were chosen randomly for evaluation. F-actin orientation (anisotropy) and expression were quantified using the open source plugin Fibriltool for Image J as described [15].

\section{Cell Migration assay and filopodia assessment}

Cell migration was carried out using Boyden Chamber assay as previously described $[2,33]$. To assess filopodia formation, RKO cells expressing control vector (EV) or TMIGD1 were transfected with moesin-GFP or control vector, GFP. Cells were fixed and pictures were taken under Nikon Deconvolution microscope equipped with camera. Quantification of filopodia was carried out using an open source software, filopodyan [40].

\section{Statistical analyses}

Experimental data were subjected to Student t-test or One-way analysis of variance, where appropriate, with representation of at least three independent experiments. $\mathrm{p}<0.05$ was considered significant, except where indicated otherwise in the figure legends. 


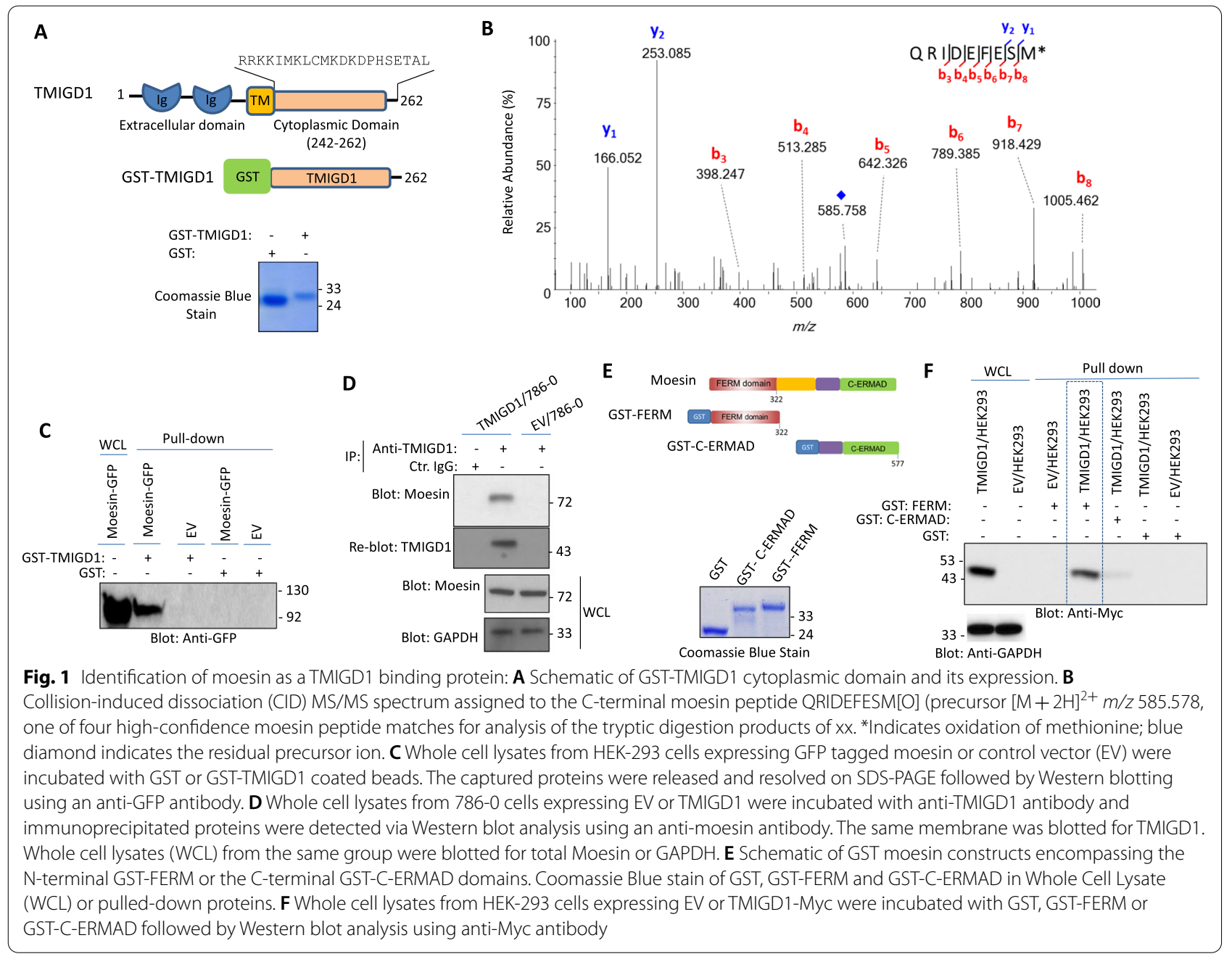

\section{Results}

Identification of moesin as a TMIGD1 Binding Protein:

To identify TMIGD1 interacting proteins in renal cancer cells, we generated a recombinant GST-fusion protein encompassing the cytoplasmic domain of human TMIGD1 (Fig. 1A). We incubated whole cell lysate derived from 786-0 renal carcinoma cells with the agarose resin bound with GST-TMIGD1 and analyzed the GST-TMIGD1 captured proteins via liquid chromatography-tandem mass spectrometry (LC-MS/MS). Moesin was one of the distinct proteins identified in these GST-TMIGD1 pull-downs (Fig. 1B). We further validated the binding of TMIGD1 with moesin in HEK293 cells ectopically expressing moesin-GFP via this same GST-TMIGD1 pull-down assay; moesin-GFP was selectively pulled-down with GST-TMIGD1 (Fig. 1C). In an alternative approach, we used 786-0 cells expressing either empty vector (EV) or TMIGD1 and determined the binding of endogenously expressed moesin with TMIGD1 in 786-0 cells via immunoprecipitation with the anti-TMIGD1 antibody. This assay also showed that moesin binds to TMIGD1 (Fig. 1D). Expression of endogenous TMIGD1 in 786-0 cells is very low/undetectable as previously reported [27].

Moesin is composed of two major domains: the $\mathrm{N}$-terminal FERM domain and the C-terminal ERMAD domain (Fig. 1E). As such, we sought to determine which of the two domains of moesin was involved in interacting with TMIGD1. To answer this question, we generated GST-FERM and GST-ERMAD fusion proteins (Fig. 1E) and evaluated their ability to interact with TMIGD1 in a GST-pulldown assay. The result showed that GSTFERM moesin selectively binds to TMIGD1 (Fig. 1F). Also noted, was a faint protein band detected with GSTERMAD-moesin, potentially suggesting a weak interaction with TMIGD1 (Fig. 1F). The data demonstrate that moesin primarily interacts with TMIGD1 via its $\mathrm{N}$-terminal FERM domain.

ERM proteins are highly conserved, possessing more than $75 \%$ amino acid homology within the common 


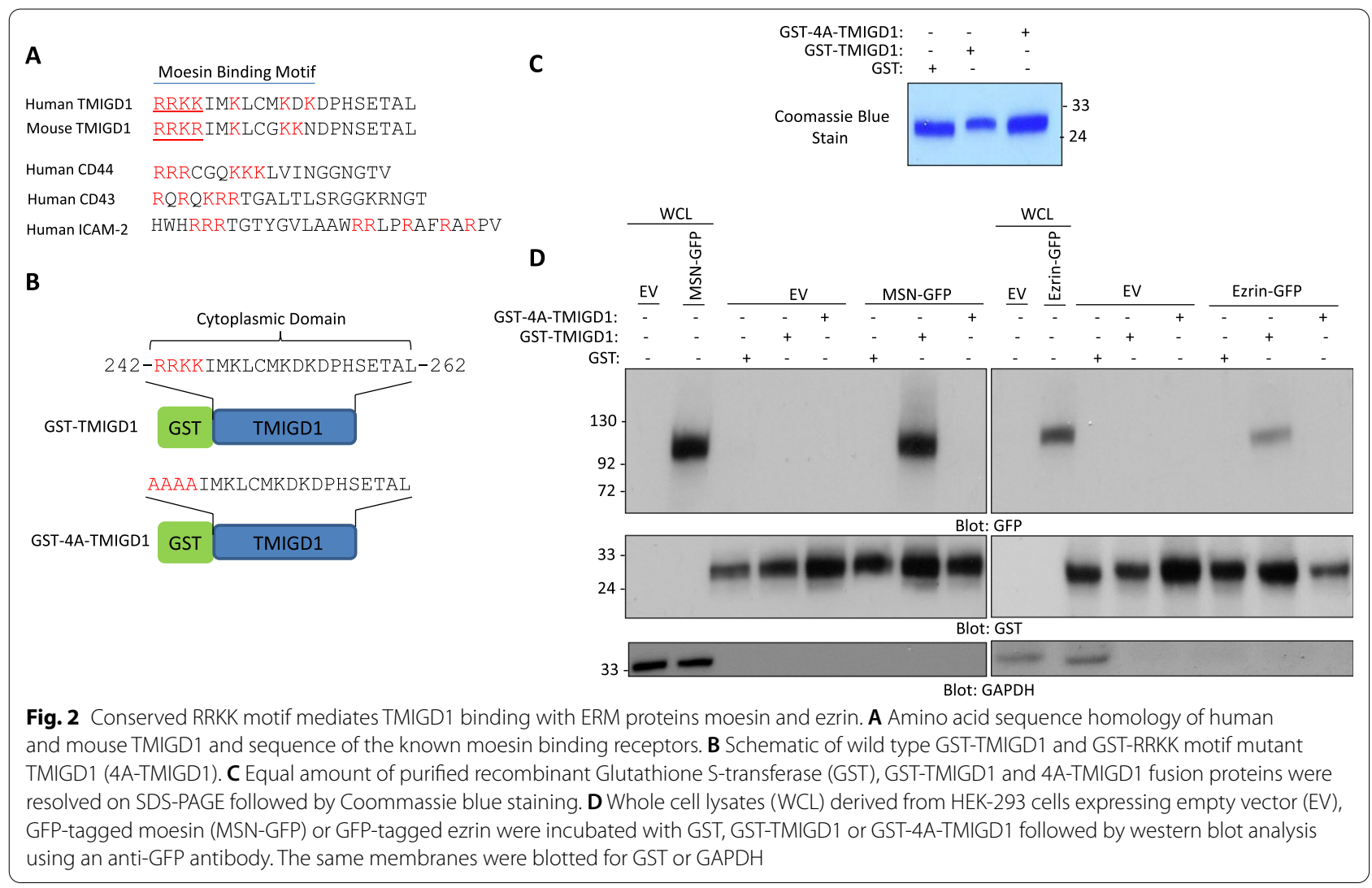

FERM domain [12]. The N-terminal FERM domain is known to recognize positively charged amino acid clusters (i.e., lysine/arginine) [43]. The amino acid sequence of moesin and ezrin are shown (Additional file 1: Figure S1). Notably, the cytoplasmic domain of TMIGD1 contains multiple positively charged amino acid clusters, which are conserved between human and mouse TMIGD1 (Fig. 2A). These positively charged amino acid clusters present on TMIGD1 have similarity to those found on CD44, CD43 and ICAM2, which are known to mediate the binding of ERM proteins [43] (Fig. 2A). These residues on TMIGD1 are thus likely to be involved in the recognition of moesin. To investigate this possibility, we generated GST-fusion TMIGD1 and GST-4ATMIGD1 proteins in which two arginine ( $R, 242$ \&243) and two lysine (K, $244 \& 245)$ residues were mutated to alanine (A) (Fig. 2B). The purified recombinant proteins were used to evaluate their interaction with moesin-GFP and ezrin-GFP expressed in HEK-293 cells (Fig. 2C). As anticipated, removal of the four positively charged residues (242-245) abolished the interaction of moesin and ezrin with TMIGD1 (Fig. 2D). Our data demonstrate that TMIGD1 interaction with ERM family proteins is established via the RRKK (242-245) motif present in the conserved C-terminus of TMIGD1.

\section{Loss of TMIGD1 in Mice Alters Apical Localization of ERM proteins Moesin and Ezrin in Epithelial cells}

ERM proteins are commonly localized at the apical membranes of polarized epithelial cells where they link filamentous (F)-actin to plasma membrane proteins $[28,34]$. Similar to the ERM proteins, TMIGD1 is also localized at the apical region of renal and intestinal epithelial cells $[2,10]$ (Fig. 3A). Our recent study demonstrated that loss of TMIGD1 in mice alters intestinal apical membrane organization [10]. Therefore, we asked whether the loss of TMIGD1 affects localization of the ERM proteins to apical membranes. Immunofluorescence staining of kidney tissues from wild type mice (TMIGD1 $+/+$ ) and TMIGD1 KO mice (TMIGD1-/-) revealed that, while moesin is largely localized to the apical membranes of renal epithelial cells in the TMIGD1+/+mouse, this apical localization and organization was decreased in TMIGD1-/mice (Fig. 3B). Genotypic analysis of TMIGD1 KO mouse used in this study is shown (Additional file 1: Figure S2). Additionally, we stained mouse intestinal tissues for ezrin, which is highly expressed in intestinal epithelial cells and required for apical membrane organization [34]. Just as we had observed for moesin in renal epithelial cells, ezrin localization at the apical 


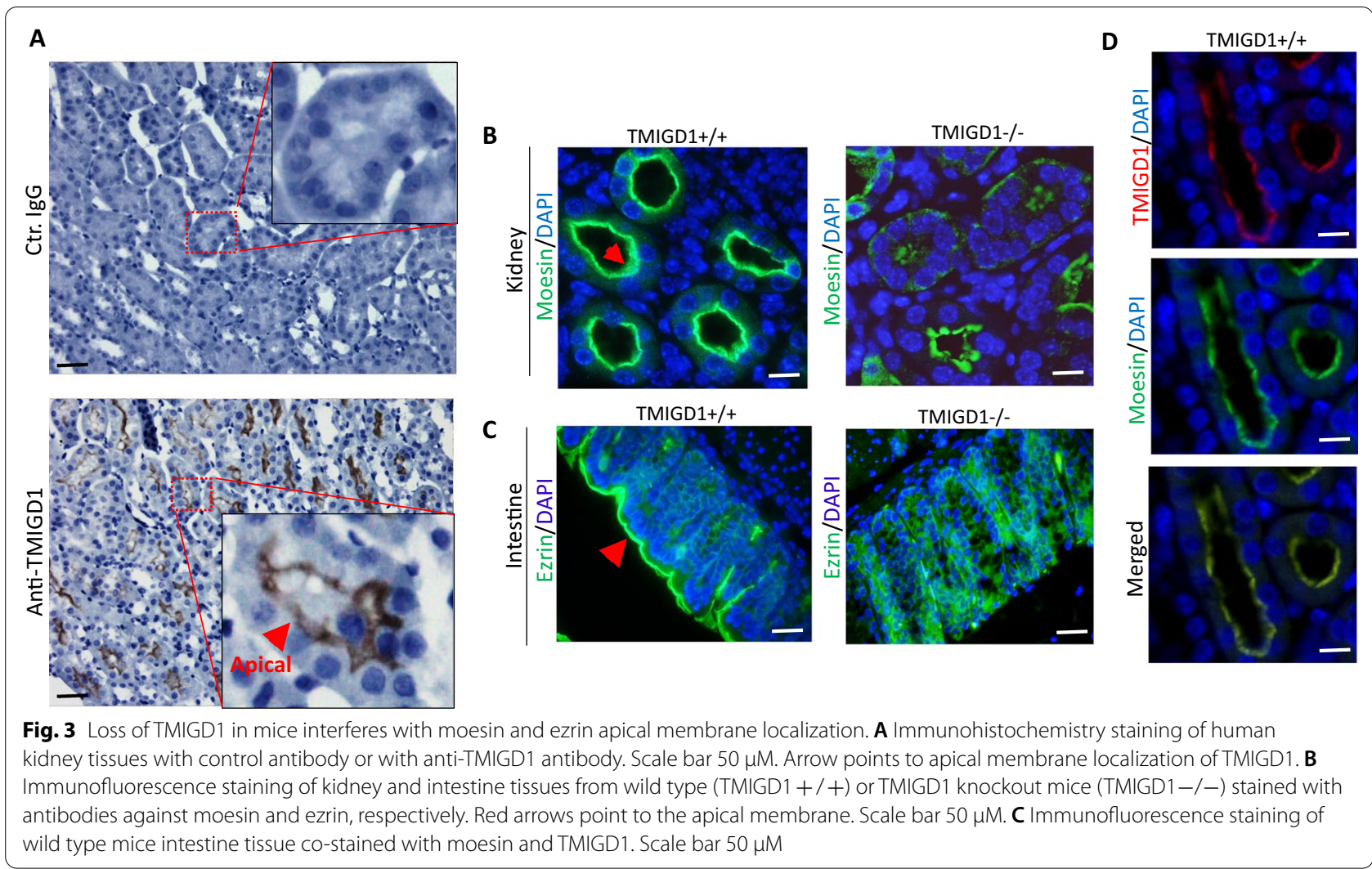

membranes in TMIGD1-/- mice was also significantly impaired (Fig. 3C).

Considering the observed binding of TMIGD1 with moesin, we also examined the co-localization of TMIGD1 with moesin. Immunofluorescence staining of mouse kidney tissue showed that TMIGD-1 co-localizes with moesin at the apical membranes of kidney epithelial cells (Fig. 3D). These data demonstrate that loss of TMIGD1 in mice interferes with the apical localization of the ERM proteins, moesin and ezrin. Moesin is highly enriched at the sites of cellular protrusions such as filopodia, microvilli and microspikes, and has been proposed to positively regulate cell migration in different cell types $[8,11,31]$. However, unlike moesin, TMIGD1 inhibits cell migration $[2,10,27]$, suggesting a potential negative regulation of moesin function by TMIGD1.

To address this question, we transfected HEK-293 cells expressing TMIGD1 or moesin with Life-ACT7 (GFP-fusion actin) and observed that HEK-293 cells expressing Life-ACT7 formed filopodia-like protrusions (Fig. 4A), whereas HEK-293 cells expressing TMIGD1 with Life-ACT7 showed inhibition of filopodia-like protrusions (Fig. 4A). Expression of TMIGD1 and moesin in HEK-293 is shown (Additional file 1: Figure S3). In an additional strategy to probe the effect of TMIGD1 in filopodia protrusions, we expressed TMIGD1 in a colorectal carcinoma cell line, RKO cells and examined filopodia structures via phalloidin staining. We chose RKO cells because they form extensive filopodia-like protrusions. Expression of TMIGD1 in RKO cells significantly inhibited filopodia protrusions (Fig. 4B). Expression of TMIGD1 in RKO cells is shown (Fig. 4B). Furthermore, we expressed moesin-GFP in RKO cells and examined moesin localization in the filopodia structures. MoesinGFP was mainly present in the filopodia structures in RKO cells (Fig. 4C). However, the presence of moesin in filopodia protrusions in the context of TMIGD1 was less prominent (Fig. 4C). Additionally, we analyzed the effect of expression of moesin in cell migration in RKO cells. The result showed that over-expression of moesin-GFP in RKO cells increased, whereas, expression of TMIGD1 inhibited cell migration (Fig. 4D). Moreover, co-expression of TMIGD1 with moesin reversed the effect of moesin in cell migration (Fig. 4D). Next, we knocked out moesin in RKO cells via CRISPR-Cas9 system and examined its effect in cell migration (Fig. 4E). Loss of moesin in RKO cells significantly reduced cell migration in RKO cells and ectopic expression of TMIGD1 in these cells further decreased cell migration (Fig. 4F). Taken together, these data demonstrate that moesin stimulates filopodialike protrusions and cell migration and TMIGD1 inhibits its pro-migratory effects in HEK-293 and RKO cells. 


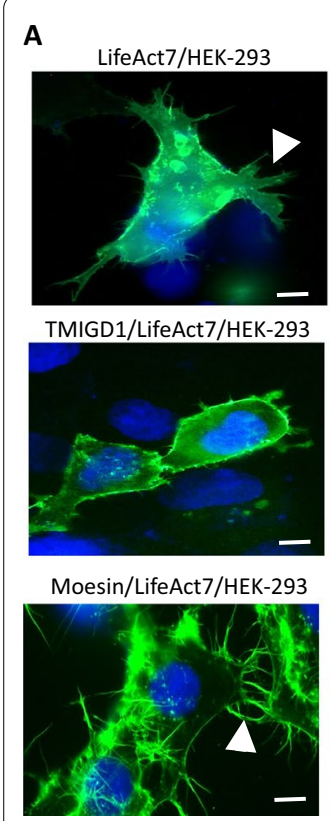

B
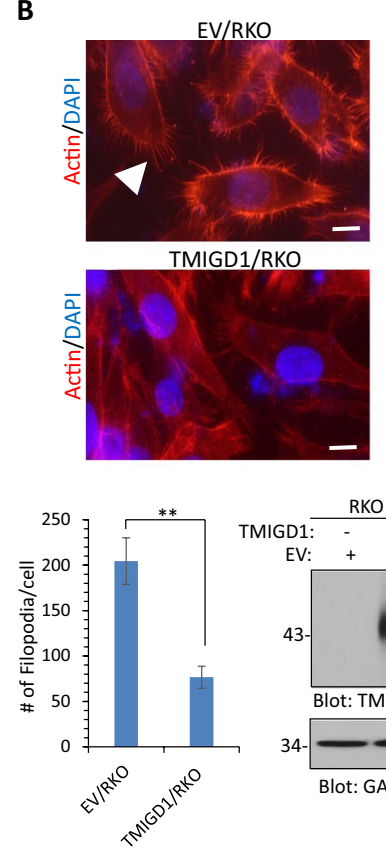

C
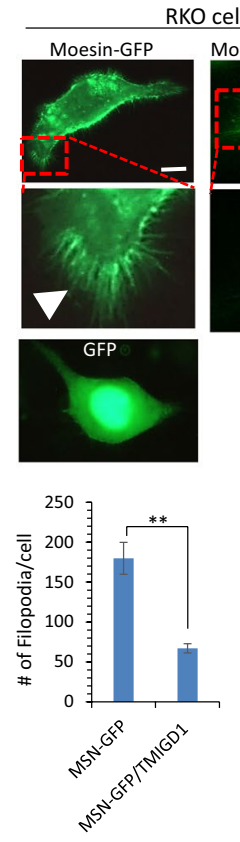

KO cells

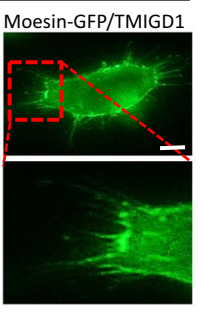

D

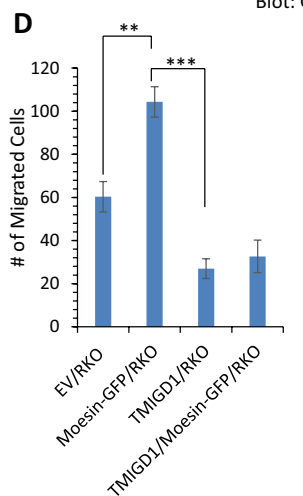

E

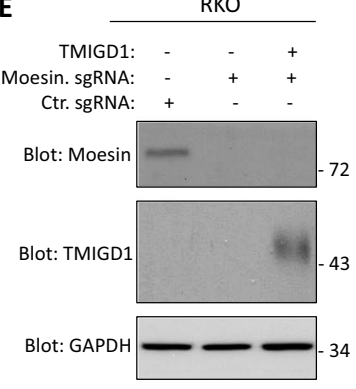

$\mathbf{F}$

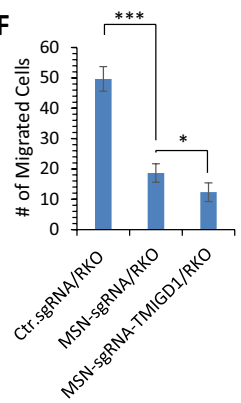

Fig. 4 Moesin stimulates and TMIGD1 inhibits filopodia protrusions and cell migration. A HEK-293 cells and HEK-293 cells expressing TMIGD1 were transfected with Life-Act7. Filopodia protrusions observed under an epifluorescence microscope after $48 \mathrm{~h}$ transfection. Image magnification, $50 \mu \mathrm{M}$. White arrows point to filopodia-like protrusions. B RKO cells expressing empty vector or TMIGD1 were stained with phalloidin for actin (red) and DAPI (nucleus). Image magnification, $50 \mu \mathrm{M}$. White arrow points to filopodia-like protrusions. The graph is a representative of filopodia formation in RKO cells expressing an empty vector (EV) or TMIGD1 (20 cells/group) ${ }^{*} p<0.01$. Cell lysates derived from RKO cells expressing empty vector or TMIGD1 were blotted for TMIGD1 and protein loading control, GAPDH. C RKO cells and RKO cells expressing TMIGD1 were transfected with moesin-GFP. Moesin-GFP localization observed under an epifluorescence microscope after $48 \mathrm{~h}$ transfection. Scale bar, $50 \mu \mathrm{M}$. The graph is a representative of filopodia formation (based on the localization of moesin-GFP) in RKO cells expressing moesin-GFP (MSN-GFP) alone or co-expressing MSN-GFP with TMIGD1. ${ }^{* *} p<0.01$. D The same cell groups were subjected to migration assay (quadruples/group) via Boyden chamber assay ${ }^{* *} p<0.01 .{ }^{* *} p<0.001$. E Western blot analysis of CRISPR-Cas9 mediated knockout of moesin in RKO cells and RKO cells expressing TMIGD1. F Cells were subjected to cell migration assay (quadruples/group) via Boyden chamber assay system * $p<0.05,{ }^{* * *} p<0.001$

\section{Expression of TMIGD1 in renal cancer cells promotes stability of microtubules}

Among all the ERM family proteins (Ezrin, Radixin and Moesin), moesin is the only known member of ERM family proteins that has been shown to bind to microtubules and regulate their stability [36]. Considering the association of TMIGD1 with moesin, we decided to investigate whether TMIGD1 expression in renal cell carcinoma, 786-0 cell line could modulate microtubule stability. First, we examined whether TMIGD1 can affect Lysine 40 (K40) acetylation of tubulin in 786-0 cells, because $K 40$ acetylation is critically important for microtubular dynamics and stability [42]. Immunofluorescence staining with an antibody against K40-acetylated tubulin in 786-0 cells expressing empty vector (EV) or TMIGD1 revealed that the level of K40-acetylated tubulin in 786-0 cells expressing TMIGD1 was significantly higher than observed for the cells expressing EV (Fig. 5A). Western blotting analysis further validated the data obtained from immunofluorescence staining analysis (Fig. 5B). Next, we asked whether expression of TMIGD1 in 786-0 cells could prevent microtubule depolymerization induced by nocodazole, a small molecule that binds to tubulin and thereby inhibits microtubule depolymerization [20, 24]. Our result showed that expression of TMIGD1 in 786-0 cells prevented nocodazole-induced microtubule depolymerization (Fig. 5C). The effect of TMIGD1 was quantified at various times after nocodazole treatment by counting the number of cells displaying intact microtubules. The most striking effect of TMIGD1 on the microtubule stability was observed at 20-30 min after nocodazole treatment (Fig. 5C). We further investigated the role of TMIGD1 in the microtubule mitotic spindle organization. Consistent with the observed effect of TMIGD1 stabilizing microtubules, 786-0 cells expressing TMIGD1 undergoing mitosis developed well-formed microtubule mitotic spindles, whereas the formation of spindles in EV/786-0 cells were relatively short and less organized 


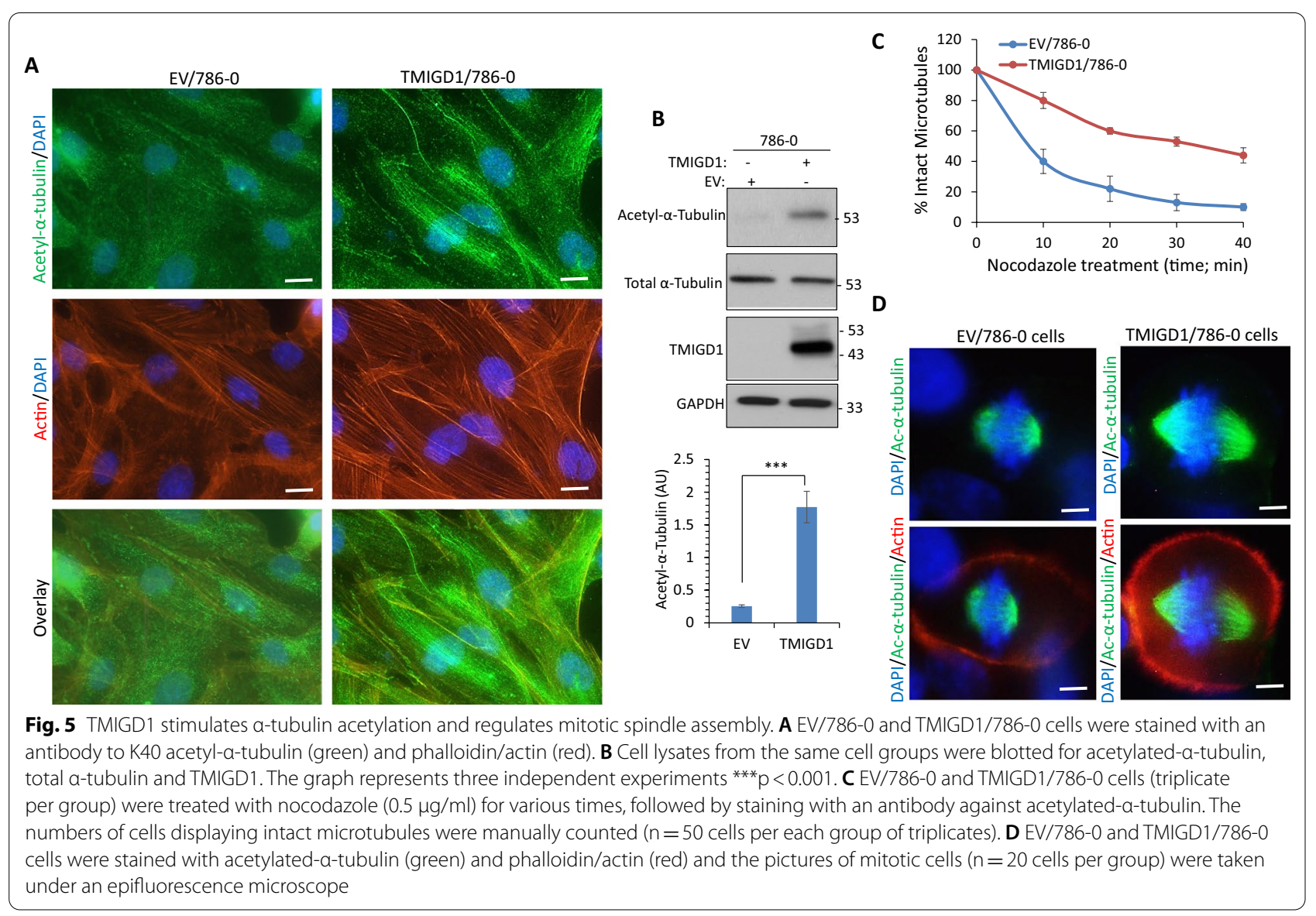

(Fig. 5D). Interestingly, there were also markedly high levels of actin on the periphery of 786-0 cells expressing TMIGD1 (Fig. 5D).

Following this finding, we asked whether inactivation of moesin via a CRISPR-Cas9 system in 786-0 cells interferes with the TMIGD1-dependent acetylation of tubulin, which is critical for microtubule mitotic spindle organization (Fig. 6A). Knockout of moesin in 786-0 cells inhibited basal acetylation (K40) of tubulin and, more importantly, markedly blocked TMIGD1dependent acetylation of tubulin (Fig. 6A). Similar effects were observed via immunofluorescence staining with anti-K40-acetyl-tubulin antibody (Fig. 6B). Considering the roles of both TMIGD1 and moesin in actin fibril organization, we examined whether the loss of moesin also affects actin fibril formation. The result showed that loss of moesin inhibited TMIGD-1 mediated F-actin orientation (Fig. 6C). Taken together, the data demonstrate that TMIGD1 dependent K40 acetylation of tubulin and F-actin orientation are mostly mediated by moesin.

\section{High expression of TMIGD1, Moesin and Ezrin correlates} with better renal cell carcinoma survival

Having established a functional link between TMIGD1 and moesin, we asked whether expression profiles of the TMIGD1 and ERM family proteins, moesin and ezrin, correlate with survival of renal cell carcinoma (RCC) patients. Our previous analysis of data sets from The Cancer Genome Atlas and our own immunohistochemical analysis revealed that low expression of TMIGD1 correlates with poor survival in renal and colon cancers $[10,27]$. Although, moesin and ezrin had been proposed to function as oncogenes in various tumor cell lines [8], their potential roles in RCC is not known. To address this question, we carried out a Kaplan-Meier survival analysis of the data set for clear cell renal cell carcinoma, the most common form of RCC, which is publicly available from The Cancer Genome Atlas via the Kaplan-Meier Plotter [29]. The result revealed that patients with RCC and high TMIGD1-, moesin- or ezrin- expressing tumors had significantly better median overall survival compared with those with low TMIGD1-expressing tumors 


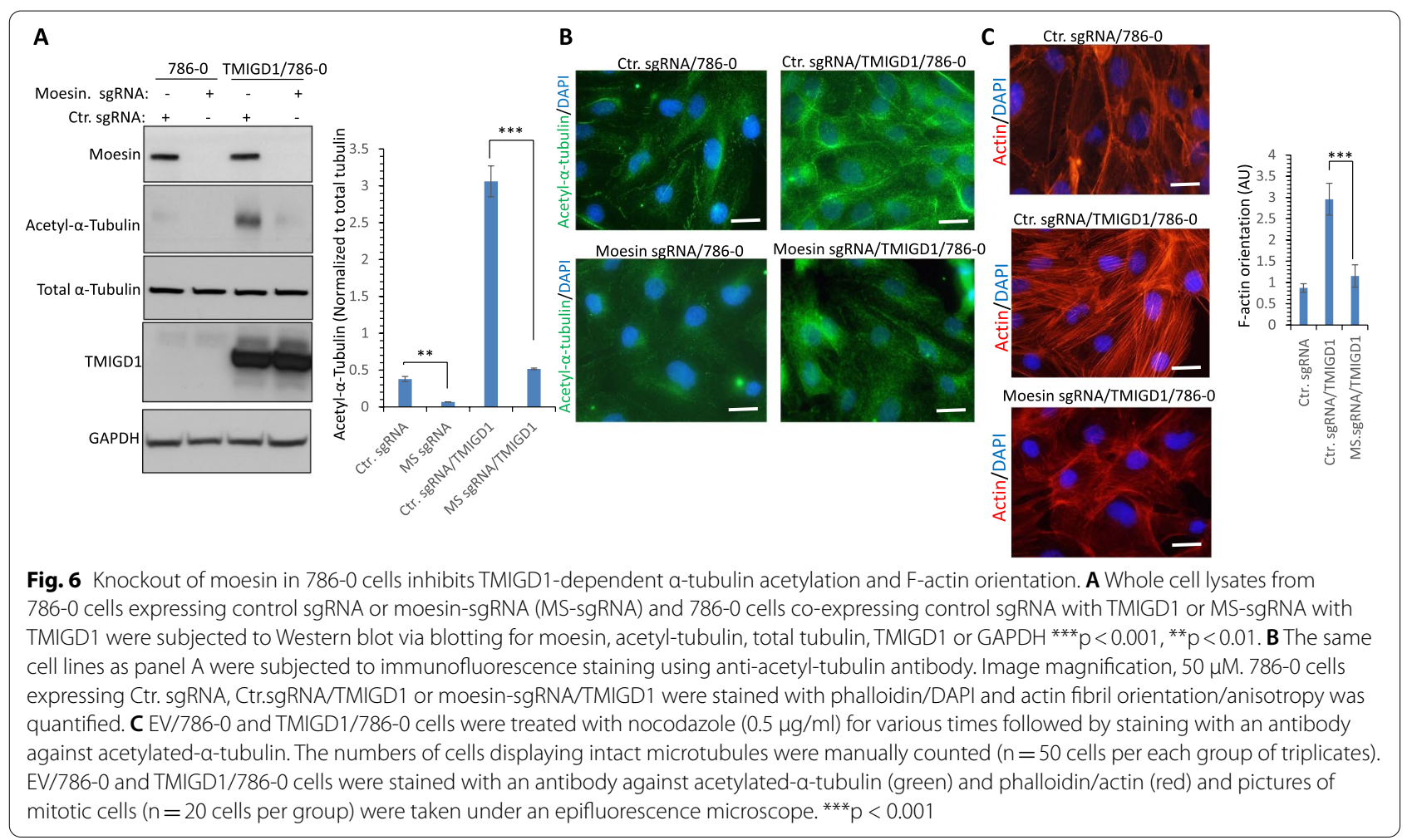

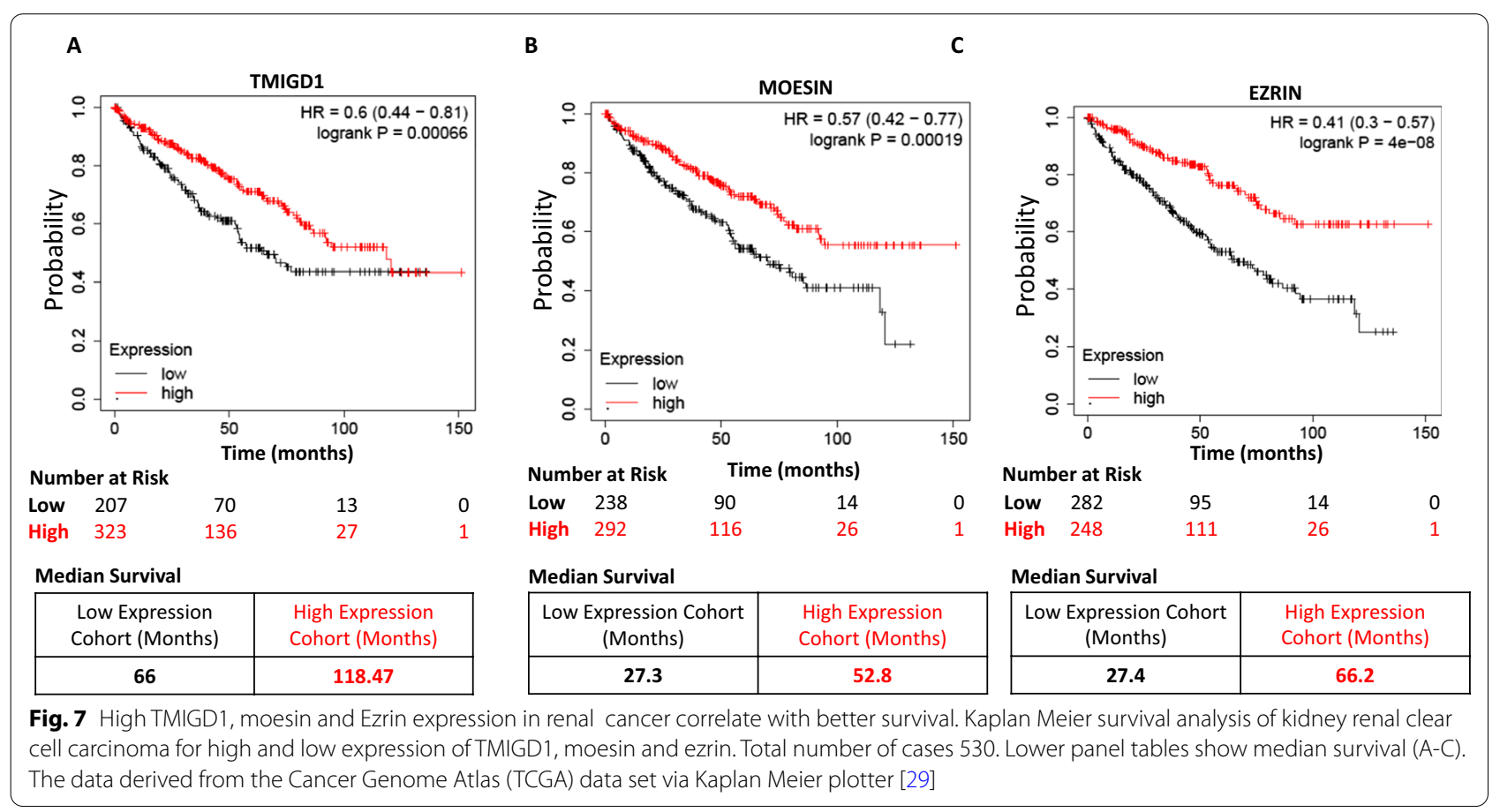


(Fig. 7A-C). The median survival for patients with tumors expressing high TMIGD1 $(\mathrm{n}=323)$ was 118.47 months versus 66 months for tumors with low TMIGD1 expression $(\mathrm{n}=207)$ (Fig. 7A). Patients with high moesinand ezrin-expressing tumors also showed significantly better survival (mean survival time for patients with tumors expressing high moesin was 52.8 months versus 27.3 months for those with low expression; the mean survival time for patients with tumors expressing high ezrin was 66.2 months versus 27.4 months for those with low expression) (Fig. 7B, C).

Having observed a correlation between better survival rate and high moesin and ezrin expression levels in human RCC, we asked whether moesin and ezrin mRNA levels are altered in RCC using the same TCGA data set via Fire Brows (http://firebrowse.org/). Our analysis demonstrated that TMIGD1 mRNA is downregulated in all the major kidney cancer types including, kidney clear cell renal cell carcinoma (KIRC), kidney papillary renal cell carcinoma (KIRP) and chromophobe renal cell carcinoma (KICH) (Additional file 1: Figure S4A). The median expression of TMIGD1 mRNA in KIRC was 2.32 versus 3.62 normal tissues, in KIRP was 1.16 versus 2.44 and in $\mathrm{KICH}$ was -1.3 versus 1.191 normal tissue (S. Figure 4A). The median expression of TMIGD1 mRNA in pan kidney cancers (KIPAN) was 1.91 versus 3.42 normal (Additional file 1: Figure S4B).

Interestingly, the expression levels of neither moesin nor ezrin were altered in a statistically significant manner in any of the kidney cancer types (Additional file 1: Figure S4B, C). The median expression of moesin mRNA in KIRC was 14.2 versus 13.7 normal tissues, in KIRP was 13.4 versus 13.3 and in $\mathrm{KICH}$ was 12.1 versus 13.4 normal tissue (Additional file 1: Figure S4B). The median expression of moesin mRNA in KIPAN was 13.6 versus 13.9 normal (Additional file 1: Figure S4B). Similarly, the median expression of ezrin mRNA in KIRC was 13.7 versus 13.4 normal tissues, in KIRP was 14.5 versus 13.5 and in $\mathrm{KICH}$ was 12.1 versus 13.4 normal tissue (Additional file 1: Figure S4C). The median expression of ezrin mRNA in KIPAN was 12.4 versus 13.4 normal (Additional file 1: Figure S4C).

Considering that we did not observe any major changes in the mRNA levels of moesin and ezrin in the human kidney cancers, we decided to analyze the TCGA data set for the presence of potential mutations on moesin and ezrin. We found no mutations on moesin or ezrin; only two or three cases of missense mutations were found (data not shown). Similarly, no significant mutations were found on TMIGD1 in RCC. There were six cases of missense and three truncating mutations. The somatic mutation frequency of TMIGD1 was $0.3 \%$ (Data not shown). Our analysis of the TCGA data demonstrating the low frequency of mutations on moesin and ezrin combined with no major alterations in their mRNA levels suggests that deregulations of cellular localization or posttranslational modifications of moesin and ezrin could account for their altered roles in tumorigenesis. Expression of TMIGD1 both at the protein levels as previously reported [10, 27] and at the mRNA levels (Additional file 1: Figure S4A) is downregulated in RCC indicating that downregulation of TMIGD1 is a primary mechanism of its alteration in human RCC, which may also account for deregulation of downstream signaling mechanisms such as signaling by the ERM family proteins moesin and ezrin in human cancer cells.

\section{Discussion}

The cell adhesion molecule TMIGD1 is a newly identified tumor suppressor that regulates critical cellular processes such as cell-cell adhesion, cell migration, cell proliferation and cell cycle [2, 10, 27]. However, the molecular mechanisms governing its signaling mechanism remains largely unknown. In this study, we identified moesin as a major TMIGD1 binding protein and a key regulator of $\alpha$-tubulin acetylation. TMIGD1 recognizes and associates with the $\mathrm{N}$-terminal FERM domain of moesin via the conserved RRKK motif located on the cytoplasmic domain and regulates moesin cellular localization. Loss of TMIGD1 in mice impairs apical membrane localization of moesin and ezrin in the epithelium of renal and intestinal tissues, respectively; cell culture studies reveal that moesin stimulates the formation of filopodia protrusions and promotes cell migration. However, co-expression of moesin with TMIGD1 hinders the pro-migratory effects of moesin. Furthermore, moesin has been proposed to regulate various cellular processes such as cell migration that are considered pro-invasion and prometastatic [8], whereas our study suggests that the role of moesin in these cellular processes is more nuanced and rather context-dependent. For example, the expression status of TMIGD1 in human cancers, such as colon and renal cancers, could determine whether moesin and ezrin function as pro- or anti-tumorigenic factors. Furthermore, our study suggests that loss of TMIGD1 in human cancers, which was previously reported in renal and colon cancers [5, 10, 27], could result in the activation of pro-tumorigenic signaling cascades, including the activation of ERM proteins that contribute to tumor progression.

Another important and interesting aspect of our study is the finding that TMIGD1 interferes with the pro-tumorigenic properties (e.g., cell migration and acetylation of tubulin) of moesin in cells perhaps through the process of binding and sequestering moesin to the apical domain of epithelial cells. Recruitment of moesin to TMIGD1 
mediates microtubular regulation, specifically aiding the K40 acetylation of $\alpha$-tubulin. The acetyltransferase aTAT1 is responsible for the $\mathrm{K} 40$ acetylation of $\alpha$-tubulin $[1,19]$ and tubulin acetylation has been shown to stimulate cell migration in multiple cell types including, fibroblasts [17], neuronal [9] and astrocytes [3], suggesting that TMIGD1/moesin pathway could regulate cell migration via modulating tubulin acetylation. Previous studies have shown that moesin connects major cytoskeletal structures to the plasma membrane and regulates cell polarity, cell adhesion and microtubule dynamics $[7,35,36]$. Since the dynamics of microtubules are altered in cancer cells and are linked to chromosomal instability and development of drug resistance $[6,18]$, they are considered an attractive target for chemotherapy against various cancer types [16]. Moesin binds directly to microtubules and regulates spindle organization in metaphase, cell morphogenesis during mitosis and cell polarity [25, 36, 37], suggesting that, by interacting with TMIGD1, moesin links TMIGD1 to microtubules and thus modulates cell polarity.

\section{Conclusions}

Identification of TMIGD1 as an upstream receptor capable of regulating the activity of ERM family proteins offers new insights into the mechanisms of TMIGD1 tumor suppressor signaling and tumorigenesis. We show for the first time that TMIGD1 binds to ERM family proteins, regulates the stability of microtubules and modulates cell migration, implicating TMIGD1 as an attractive potential cancer therapeutic target. Further investigation into mechanisms by which TMIGD1 regulates moesin cellular localization and activity should provide an increasingly mechanistic explanation of the interplay between TMIGD1 and ERM family proteins. Furthermore, considering our observation that higher expression profiles of TMIGD1, moesin and ezrin correlate with better survival, deeper investigation into the molecular basis of downregulation of TMIGD1, moesin and ezrin will shed new insight into the altered roles of cell adhesion signaling mechanisms in tumor progression.

\section{Supplementary Information}

The online version contains supplementary material available at https://doi. org/10.1186/s12929-021-00757-z.

Additional file 1: Figure S1. Amino acid sequence homology of human moesin and ezrin. Alignment of human moesin (MSN) and ezrin (EZN) is shown. The FERM domain is shown in a box. Hydrophobic and negatively amino acids are highlighted in light blue and light brown colors, respectively. Figure S2. qPCR analysis of TMIGD1 KO mouse: qPCR analysis of wild-type and TMIGD1 is shown. Figure S3. Ectopic expression of Moesin and TMIGD1 in HEK-293 cell. Whole cell lysates derived from HEK-293 cells expressing TMIGD1 or moesin subjected to western blot analysis. Figure S4. TMIGD1, Moesin and Ezrin mRNA levels in human normal kidney verses kidney cancer types. A-C TMIGD1, Moesin and Ezrin mRNA levels obtained from the TCGA data set via Fire Brows (http://firebrowse.org/). Kidney cancer types; kidney clear cell renal cell carcinoma (KIRC), kidney papillary renal cell carcinoma (KIRP), chromophobe renal cell carcinoma (KICH) and kidney pan cancer analysis (KIPAN). The horizontal small black bars within each box correspond to median expression of given proteins.

Acknowledgements

Not applicable.

Authors' contributions

NR, CEC and RX-YO were involved in writing and editing of the manuscript. NR, $\mathrm{KBC}, \mathrm{KCSDC}, \mathrm{RA}, \mathrm{AM}$ and NE all were involved in the design, performing the experiments. All authors read and approved the final manuscript.

Funding

This work was supported in part through grants from CTSI grant

(UL1TR001430) and Malory Fund, Department of Pathology, Boston University (NR), P41 GM104603 (CEC) and R24 GM134210 (CEC).

Availability of data and materials

Cell lines, plasmids and other reagents described in this manuscript are available upon a reasonable request.

\section{Declarations}

Ethics approval and consent to participate

The care and use of laboratory animals were approved by Boston University.

Consent for publication

Not applicable.

Competing interests

Authors declare no competing interest.

Received: 14 May 2021 Accepted: 27 August 2021

Published online: 09 September 2021

\section{References}

1. Akella JS, Wloga D, Kim J, Starostina NG, Lyons-Abbott S, Morrissette NS, Dougan ST, Kipreos ET, Gaertig J. MEC-17 is an alpha-tubulin acetyltransferase. Nature. 2010;467(7312):218-22

2. Arafa E, Bondzie PA, Rezazadeh K, Meyer RD, Hartsough E, Henderson JM, Schwartz JH, Chitalia V, Rahimi N. TMIGD1 is a novel adhesion molecule that protects epithelial cells from oxidative cell injury. Am J Pathol. 2015;185(10):2757-67.

3. Bance B, Seetharaman S, Leduc C, Boeda B, Etienne-Manneville S. Microtubule acetylation but not detyrosination promotes focal adhesion dynamics and astrocyte migration. J Cell Sci. 2019. https://doi.org/10. 1242/jcs.225805.

4. Baumgartner M, Sillman AL, Blackwood EM, Srivastava J, Madson N, Schilling JW, Wright JH, Barber DL. The Nck-interacting kinase phosphorylates ERM proteins for formation of lamellipodium by growth factors. Proc Natl Acad Sci U S A. 2006;103(36):13391-6.

5. Cattaneo E, Laczko E, Buffoli F, Zorzi F, Bianco MA, Menigatti M, Bartosova Z, Haider R, Helmchen B, Sabates-Bellver J, Tiwari A, Jiricny J, Marra G. Preinvasive colorectal lesion transcriptomes correlate with endoscopic morphology (polypoid vs. nonpolypoid). EMBO Mol Med. 2011;3(6):334-47.

6. Cirillo L, Gotta M, Meraldi P. The elephant in the room: the role of microtubules in cancer. Adv Exp Med Biol. 2017;1002:93-124.

7. Clucas J, Valderrama F. ERM proteins in cancer progression. J Cell Sci. 2015;128(6):1253.

8. Clucas J, Valderrama F. ERM proteins in cancer progression. J Cell Sci. 2014;127(Pt 2):267-75.

9. Creppe C, Malinouskaya L, Volvert ML, Gillard M, Close P, Malaise O, Laguesse S, Cornez I, Rahmouni S, Ormenese S, Belachew S, Malgrange 
B, Chapelle JP, Siebenlist U, Moonen G, Chariot A, Nguyen L. Elongator controls the migration and differentiation of cortical neurons through acetylation of alpha-tubulin. Cell. 2009;136(3):551-64.

10. De La Cena KOC, Ho RX, Amraei R, Woolf N, Tashjian JY, Zhao Q, Richards S, Walker J, Huang J, Chitalia VC, Rahimi N. Transmembrane and Immunoglobulin Domain Containing 1, a Putative Tumor Suppressor, Induces G2/M Cell Cycle Checkpoint Arrest in Colon Cancer Cells. Am J Pathol. 2021;191(1):157-67.

11. DeSouza LV, Matta A, Karim Z, Mukherjee J, Wang XS, Krakovska O, Zadeh G, Guha A, Siu KM. Role of moesin in hyaluronan induced cell migration in glioblastoma multiforme. Mol Cancer. 2013;12:74.

12. Gary R, Bretscher A. Ezrin self-association involves binding of an N-terminal domain to a normally masked C-terminal domain that includes the F-actin binding site. Mol Biol Cell. 1995;6(8):1061-75.

13. Giles RH, Voest EE. Tumor suppressors APC and VHL: gatekeepers of the intestine and kidney. Prog Mol Subcell Biol. 2005:40:151-81.

14. Halaoui R, McCaffrey L. Rewiring cell polarity signaling in cancer. Oncogene. 2015;34(8):939-50.

15. Ho RX, Tahboub R, Amraei R, Meyer RD, Varongchayakul N, Grinstaff M, Rahimi N. The cell adhesion molecule IGPR-1 is activated by and regulates responses of endothelial cells to shear stress. J Biol Chem. 2019;294(37):13671-80

16. Honore S, Pasquier E, Braguer D. Understanding microtubule dynamics for improved cancer therapy. Cell Mol Life Sci. 2005;62(24):3039-56.

17. Hubbert C, Guardiola A, Shao R, Kawaguchi Y, Ito A, Nixon A, Yoshida M, Wang XF, Yao TP. HDAC6 is a microtubule-associated deacetylase. Nature. 2002:417(6887):455-8.

18. Jordan MA, Wilson L. Microtubules as a target for anticancer drugs. Nat Rev Cancer. 2004;4(4):253-65.

19. Kalebic N, Sorrentino S, Perlas E, Bolasco G, Martinez C, Heppenstall PA. alphaTAT1 is the major alpha-tubulin acetyltransferase in mice. Nat Commun. 2013;4:1962

20. Kaur R, Kaur G, Gill RK, Soni R, Bariwal J. Recent developments in tubulin polymerization inhibitors: an overview. Eur J Med Chem. 2014;87:89-124.

21. Lamb RF, Ozanne BW, Roy C, McGarry L, Stipp C, Mangeat P, Jay DG Essential functions of ezrin in maintenance of cell shape and lamellipodial extension in normal and transformed fibroblasts. Curr Biol. 1997:7(9):682-8

22. Lan M, Kojima T, Murata M, Osanai M, Takano K, Chiba H, Sawada N. Phosphorylation of ezrin enhances microvillus length via a p38 MAPkinase pathway in an immortalized mouse hepatic cell line. Exp Cell Res. 2006:312(2):111-20

23. Lee M, Vasioukhin V. Cell polarity and cancer-cell and tissue polarity as a non-canonical tumor suppressor. J Cell Sci. 2008:121(Pt 8):1141-50.

24. Lu Y, Chen J, Xiao M, Li W, Miller DD. An overview of tubulin inhibitors that interact with the colchicine binding site. Pharm Res. 2012;29(11):2943-71.

25. Medina E, Williams J, Klipfell E, Zarnescu D, Thomas G, Le Bivic A. Crumbs interacts with moesin and beta(Heavy)-spectrin in the apical membrane skeleton of Drosophila. J Cell Biol. 2002;158(5):941-51.

26. Meyer RD, Srinivasan S, Singh AJ, Mahoney JE, Gharahassanlou KR, Rahimi N. PEST motif serine and tyrosine phosphorylation controls vascular endothelial growth factor receptor 2 stability and downregulation. Mol Cell Biol. 2011:31(10):2010-25

27. Meyer RD, Zou X, Ali M, Ersoy E, Bondzie PA, Lavaei M, Alexandrov I, Henderson J, Rahimi N. TMIGD1 acts as a tumor suppressor through regulation of p21Cip1/p27Kip1 in renal cancer. Oncotarget. 2018;9(11):9672-84.
28. Morales FC, Takahashi Y, Kreimann EL, Georgescu MM. Ezrin-radixinmoesin (ERM)-binding phosphoprotein 50 organizes ERM proteins at the apical membrane of polarized epithelia. Proc Natl Acad Sci U S A. 2004;101(51):17705-10

29. Nagy A, Munkacsy G, Gyorffy B. Pancancer survival analysis of cancer hallmark genes. Sci Rep. 2021;11(1):6047.

30. Nogales E. Structural insight into microtubule function. Annu Rev Biophys Biomol Struct. 2001;30:397-420.

31. Okayama T, Kikuchi S, Ochiai T, Ikoma H, Kubota T, Ichikawa D, Fujiwara H, Okamoto K, Sakakura C, Sonoyama T, Kokuba Y, Doi Y, Tsukita S, Bissell DM Otsuji E. Attenuated response to liver injury in moesin-deficient mice: impaired stellate cell migration and decreased fibrosis. Biochim Biophys Acta. 2008;1782(9):542-8.

32. Rahimi $N$, Dayanir $V$, Lashkari K. Receptor chimeras indicate that the vascular endothelial growth factor receptor-1 (VEGFR-1) modulates mitogenic activity of VEGFR-2 in endothelial cells. J Biol Chem. 2000;275(22):16986-92.

33. Rahimi N, Rezazadeh K, Mahoney JE, Hartsough E, Meyer RD. Identification of IGPR-1 as a novel adhesion molecule involved in angiogenesis. Mol Biol Cell. 2012;23(9):1646-56.

34. Saotome I, Curto M, McClatchey Al. Ezrin is essential for epithelial organization and villus morphogenesis in the developing intestine. Dev Cell. 2004;6(6):855-64.

35. Sauvanet C, Wayt J, Pelaseyed T, Bretscher A. Structure, regulation, and functional diversity of microvilli on the apical domain of epithelial cells. Annu Rev Cell Dev Biol. 2015;31:593-621.

36. Solinet S, Mahmud K, Stewman SF, El Ben Kadhi K, Decelle B, Talje L, Ma A Kwok BH, Carreno S. The actin-binding ERM protein Moesin binds to and stabilizes microtubules at the cell cortex. J Cell Biol. 2013;202(2):251-60.

37. Speck O, Hughes SC, Noren NK, Kulikauskas RM, Fehon RG. Moesin functions antagonistically to the Rho pathway to maintain epithelial integrity. Nature. 2003;421(6918):83-7.

38. Takeuchi K, Sato N, Kasahara H, Funayama N, Nagafuchi A, Yonemura S, Tsukita S, Tsukita S. Perturbation of cell adhesion and microvilli formation by antisense oligonucleotides to ERM family members. J Cell Biol. 1994;125(6):1371-84.

39. Thiery JP. Epithelial-mesenchymal transitions in tumour progression. Nat Rev Cancer. 2002;2(6):442-54.

40. Urbancic V, Butler R, Richier B, Peter M, Mason J, Livesey FJ, Holt CE, Gallop JL. Filopodyan: an open-source pipeline for the analysis of filopodia. J Cell Biol. 2017;216(10):3405-22.

41. Vilmos P, Kristo I, Szikora S, Jankovics F, Lukacsovich T, Kari B, Erdelyi M. The actin-binding ERM protein Moesin directly regulates spindle assembly and function during mitosis. Cell Biol Int. 2016;40(6):696-707.

42. Xiao H, Verdier-Pinard P, Fernandez-Fuentes N, Burd B, Angeletti R, Fiser A, Horwitz SB, Orr GA. Insights into the mechanism of microtubule stabilization by Taxol. Proc Natl Acad Sci U S A. 2006;103(27):10166-73.

43. Yonemura S, Hirao M, Doi Y, Takahashi N, Kondo T, Tsukita S, Tsukita S. Ezrin/radixin/moesin (ERM) proteins bind to a positively charged amino acid cluster in the juxta-membrane cytoplasmic domain of CD44, CD43, and ICAM-2. J Cell Biol. 1998;140(4):885-95.

\section{Publisher's Note}

Springer Nature remains neutral with regard to jurisdictional claims in published maps and institutional affiliations. 